Capturing the essence of a metabolic network: $A$ flux balance analysis approach

Murabito, Ettore and Simeonidis, Evangelos and

Smallbone, Kieran and Swinton, Jonathan

2009

MIMS EPrint: 2009.42

Manchester Institute for Mathematical Sciences

School of Mathematics

The University of Manchester

\footnotetext{
Reports available from: http://eprints.maths.manchester.ac.uk/

And by contacting: The MIMS Secretary

School of Mathematics

The University of Manchester

Manchester, M13 9PL, UK
} 


\section{Capturing the essence of a metabolic network: A Flux Balance Analysis approach}

Ettore Murabito ${ }^{\mathrm{a}, \mathrm{b}}$, Evangelos Simeonidis, ${ }^{\mathrm{b}, \mathrm{c}}$, Kieran Smallbone ${ }^{\mathrm{c}, \mathrm{d}}$, Jonathan Swinton ${ }^{\mathrm{e}}$

a. Doctoral Training Centre Integrative Systems Biology from Molecules to Life,

Manchester Interdisciplinary Biocentre, The University of Manchester, 131 Princess

Street, Manchester M1 7DN, UK.

b. School of Chemical Engineering and Analytical Science, The University of Manchester, Sackville Street, Manchester M60 1QD, UK.

c. Manchester Centre for Integrative Systems Biology, Manchester Interdisciplinary

Biocentre, The University of Manchester, 131 Princess Street, Manchester M1 7DN, UK.

d. School of Mathematics, The University of Manchester, Oxford Road, Manchester M13 9PL, UK.

e. Computational Biology/Advanced Science and Technology Laboratory, AstraZeneca.

Corresponding author: Ettore Murabito (email: ettore.murabito@postgrad.manchester.ac.uk

- phone: +44 (0) 1613065146 - fax: +44 (0) 16130 65201) 


\begin{abstract}
As genome-scale metabolic reconstructions emerge, tools to manage their size and complexity will be increasingly important. Flux Balance Analysis (FBA) is a constraint-based approach widely used to study the metabolic capabilities of cellular or subcellular systems. FBA problems are highly underdetermined and many different phenotypes can satisfy any set of constraints through which the metabolic system is represented.

Two of the main concerns in FBA are exploring the space of solutions for a given metabolic network and finding a specific phenotype which is representative for a given task such as maximal growth rate. Here we introduce a recursive algorithm suitable for overcoming both of these concerns. The method proposed is able to find the alternate optimal patterns of active reactions of a FBA problem and identify the minimal subnetwork able to perform a specific task as optimally as the whole. Our method represents an alternative to and an extension of other approaches conceived for exploring the space of solutions of an FBA problem. It may also be particularly helpful in defining a scaffold of reactions upon which to build up a dynamic model, when the important pathways of the system have not yet been welldefined.
\end{abstract}

Keywords: Mixed Integer Linear Programming problem; alternate optimal solutions; alternate optimal patterns; minimal optimal subnetwork; minimal effort principle. 


\section{Introduction}

FBA is a constraint-based approach suitable for studying the range of possible phenotypes of a metabolic system (Edwards and Palsson, 1999; Edwards and Palsson, 2002; Förster et al., 2003; Kauffman et al., 2003; Varma et al., 1993). Despite this declared intent, it is common practice to rely on one single optimal solution (usually the first one found) for further studies on the system under investigation (Fell and Small, 1986; Ramakrishna et al., 2001; Savinell and Palsson, 1992), even though it is well-known that FBA problems generally have many more than one equivalent solutions that all satisfy the optimality criteria - a behaviour of the system that is known in optimisation as degeneracy. Available software packages and optimisation solvers do not typically find alternate optima, but stop after the first solution is found. This solution is then treated as representative of the pattern of flux in the real system, but no computational or biological evidence actually supports this arbitrary choice. In order to make the simulated phenotype more representative of reality, a common approach is to reduce the feasible space of solutions by enriching the system with additional information (i.e. constraints) retrieved from experimental measurements (Bonarius et al., 1996; Llaneras and Picó, 2008; Varma and Palsson, 1994). These may refer, for example, to the maximum activity of the enzymes catalysing the different reactions in the network or even to the current value of certain fluxes under the conditions considered. Moreover, in order to guarantee the feasibility of the solutions, reaction thermodynamics is often included in FBA by imposing additional non-linear constraints describing energy balance with the chemical potential (Beard et al., 2002; Beard et al., 2004).

In the original formulation of FBA, only topological and stoichiometric information is considered, while the dynamic aspects of the system are neglected. The topology and stoichiometry of a metabolic network can be represented through the stoichiometric 
matrix $\mathbf{S}$, whose generic element $s_{i j}$ is the stoichiometric coefficient of metabolite $i$ in reaction $j$ (Lee et al., 2006). The mass balance equations at steady-state for all the metabolites can then be expressed through the following expression

$$
\mathbf{S} \cdot \mathbf{v}=\mathbf{0}
$$

where $\mathbf{v}$ is the flux vector whose elements $v_{j}$ represent the rate of each reaction $j$ in the network. Additional constraints can also be added in order to bind the fluxes within a certain range of values

$$
v_{j}^{L} \leq v_{j} \leq v_{j}^{U} \quad j=1,2, \ldots, n
$$

where $v_{j}^{L}$ and $v_{j}^{U}$ are lower and upper bounds respectively for fluxes $v_{j}$. These constraints are generally used to determine the reversibility of the reactions as well as to establish a maximum value for their flux when the corresponding $V_{\max }$ has been measured experimentally, or to specify the availability of specific nutrients (Förster et al., 2003; Schilling et al., 2002; Varma and Palsson, 1994). The polytope defined by the set of constraints (1) and (2) is called the space of feasible solutions or, more simply, the feasible space.

The main concern in FBA is finding, within the feasible space of solutions, the flux distributions that allow the system to optimally perform a specific task (e.g. maximization of biomass production). The general formulation of a FBA problem may then be expressed as follows

$$
\begin{gathered}
\max f(\mathbf{v}) \\
f: \mathbf{R}^{n} \rightarrow \mathbf{R} \\
\text { subj ect to }
\end{gathered}
$$

(1), (2)

where $f$ is a mathematical function, called objective function, representing the response of the system in terms of its ability to perform the task we are interested in. The aim is then 
to identify feasible sets of steady-state fluxes optimizing the stated cellular function represented by $f$, for a metabolic network subject to a set of mass conservation constraints such as (1) (Price et al., 2004).

The objective function $f$ is usually represented by a linear combination of flux variables $v_{j}$

$$
f=\sum_{j=1}^{n} \alpha_{j} v_{j}
$$

This, together with the linearity of constraints (1) and (2), casts the general formulation of FBA into a linear programming (LP) problem.

\section{Alternate optimal solutions and alternate optimal patterns}

FBA problems are in general strongly underdetermined. The underlying assumption of the use of a linear objective function, in fact, implies that its level sets are hyperplanes and that the level set corresponding to the optima may be parallel to one of the edges or facets of the convex polytope defined by constraints (1) and (2). Although this leads to an infinite number of optimal solutions, we are often interested in a finite subset of them, where each solution differs from the others in the set of its active reactions (or non null vectorial components). More precisely, we are interested in finding all the different patterns of active reactions that are compatible with the criterion of optimality of an FBA problem. This set of optimal patterns is in part identified by the so called alternate optimal solutions, represented by the extreme points that lie at the intersection between the hyperplane of the objective function and the polytope of the feasible space (Lee et al., 2000), but is not limited to them. The optimal patterns, in fact, can also be partly derived through overpositions of different alternate optima solutions. Given the similarity of these two concepts, from now on we will refer to the set of optimal patterns as alternate optimal patterns. 


\section{The principle of minimal effort}

It is not a trivial task to find all the alternate optimal patterns, nor to assess which one is best at representing the real pattern of fluxes in the cell under the specific conditions considered.

Attempts in addressing these two issues have been proposed separately. The literature reports a number of algorithms for finding the extreme points of the convex polytope defined by equations (1-2) (Matheiss and Rubin, 1980; Swart, 1985). Lee et al., in particular, proposed a recursive method for finding the alternate optimal solutions in linear programming (LP) models for metabolic networks (Lee et al., 2000). On the other hand, different ways have been proposed to find solutions representative of the system under examination (Holzhütter, 2004; León et al., 2008; Smallbone and Simeonidis, 2009). Holzhütter (2004), in particular, suggested an approach based on the principle of "minimal effort" to infer the solution most likely to represent the true pattern of fluxes. The algorithm presented in this paper is suitable for both these purposes, although the principle of minimal effort has been formulated differently. Here the set of active reactions is minimized rather than the total flux through the network (as in Holzhütter's work), hence the problem is turned into finding the minimal subnetwork with the same optimal capabilities as the whole network.

The biological rationale behind this approach can be explained as follows: given a specific cellular function to accomplish and given a set of available external substrates, the cell should minimize the number of enzymes necessary to optimally perform this function. This formulation of the principle of minimal effort turns to be particularly suitable when the cellular function to optimize is the production of biomass. There is experimental evidence that decrease in the number of active reaction steps and increase in growth rate can occur in parallel. More specifically, turning off reactions that do not contribute to growth, allows nutrients and proteins that were used in these processes to be 
"reallocated" and used to produce biomass (Acerenza and Graña, 2006; Cooper and Lenski, 2000; Graña and Acerenza, 2001).

A more practical reason for applying the principle of minimal effort as stated above is that the minimal optimal solution provides a concise set of reactions that can be used as a backbone for the development of kinetic models which capture the essence of the whole system, being at the same time as small as possible. This would allow researchers to build large-scale kinetic models of organisms, by providing an idea about which pathways can be reasonably neglected.

To illustrate the algorithm, two examples are presented. First, a "toy model" is used as a workbench to assess the suitability of the algorithm. Then, a simplified reconstruction of the central carbon metabolism of $E$. Coli is used to compare the results between our algorithm and the one presented by Lee et al. (Lee et al., 2000). The detailed formulation of the algorithm is given in the Methods section.

\section{Results}

\section{A toy network}

The "toy model" used in this first example (Figure 1) has been conceived to easily assess the suitability of the algorithm for the aims it addresses. The network consists of 16 reactions and 10 compounds. All fluxes are bound between -10 and 10 (arbitrary units), except for reaction 1 where the lower bound is set to 0 .

The criterion of optimality is the maximization of the flux through reaction 16 , which may be seen as the output of the system. By applying our algorithm, 109 alternate optimal patterns were found, with an optimum value of $v_{16}=1$. 
Some of these patterns are shown graphically in Figure 2. It is easy to see how they can be decomposed into a linear pathway, connecting the input $\left(v_{1}\right)$ and the output $\left(v_{16}\right)$ of the system, and one or more loops of reactions. These loops are referred as infeasible cycles because they can carry a non-null flux without any communication between the system and the external environment in terms of exchange of matter (Schilling et al., 2000). The presence of infeasible cycles is one of the major causes of degeneracy in an FBA problem. In order to prevent the optimal solutions containing any infeasible cycles, reaction thermodynamics is sometimes included in FBA by imposing additional non-linear constraints describing energy balance with the chemical potential (Beard et al., 2002; Beard et al., 2004). The infeasibility of these reaction loops makes them not only biophysically unachievable but also unnecessary for the optimality of the system. Consequently, in minimal optimal solutions (i.e. solutions satisfying the previous criterion of optimality and having the minimum possible number of active reactions) no infeasible cycles can be present.

In order to find these solutions, and hence their corresponding pattern of active reactions, we fixed the flux of reaction 16 at the optimal value identified previously and we run the algorithm with a new criterion of optimality: minimizing the number of non-zero fluxes. The algorithm found 8 minimal alternate optimal patterns consisting of 6 active reactions each (Figure 3). Thanks to the simple topology and the symmetry of this network it is easy to see that a minimal solution cannot have less than 6 active reactions and that there are no minimal patterns other than the eight identified, providing evidence of the suitability of the algorithm for finding all the alternate optimal patterns of a linear optimization problem and its minimal solutions. 


\section{A model of the central carbon metabolism in E. Coli.}

After testing the algorithm on the toy model, we applied it on a simplified reconstruction of the central carbon metabolism of E. Coli (Figure 4). This model is taken from Lee et al (Lee et al., 2000) in a study aimed to identify the alternatives in the carbon trafficking in mutants lacking pyruvate kinase. The model consists of 30 metabolites and 34 reactions or, in terms of the stoichiometry matrix, 30 equations (mass balances) and 34 variables. The complete set of constraints used in Lee et al. (balance equations, lower and upper bounds) is reported in the Appendix. Since some of the reactions are supposed to have a constant flux (equations a.17-a.27 of Appendix) it is possible to remove them from the set of variables and deal with a reduced system consisting of 18 equations and 23 variables. Our algorithm was run to find the alternate optimal patterns while minimizing the flux through reaction 18 (pyruvate kinase). The algorithm found 28 alternate optimal patterns - versus the 9 alternate optimal solutions obtained by Lee et al. Although Lee's approach has a slightly different scope than ours (rather than finding alternate optimal patterns it finds alternate optimal solutions, which are a subset of the former), these results show an improvement in respect to similar approaches previously proposed to explore the feasible space. The complete set of optimal patterns is presented in Table 1.

We also applied our algorithm to find the minimal optimal solution(s) of the system. To do so, we fixed the flux of reaction 18 to the optimal (minimal) value found previously (this ensures the optimality of the minimal solutions we wanted to find) and we minimized, as objective function, the number of active reactions.

The algorithm found one minimal solution consisting of 27 active reactions (including the reactions with fixed flux). The solution is represented in Table 1 by the highlighted column. 


\section{Discussion}

Even though FBA is meant in principle to provide insights on the whole range of possibilities for a metabolic network rather than identifying a specific phenotype, it is common practice to rely only on one specific solution for further studies and analysis (Ramakrishna et al., 2001; Savinell and Palsson, 1992). Sometimes different solutions are found by changing the constraints on the flux variables in order to simulate, for example, different growth conditions (Edwards and Palsson, 2000; León et al., 2008). This leads de facto to a different FBA problem and doesn't answer the question about how many alternative ways the system has to optimally achieve a specific task in a given condition specified by a fixed set of constraints.

Finding a way to explore more extensively the range of possibilities of a metabolic network and identifying a pattern of fluxes which can reasonably represent the real trafficking of matter in the system are two of the main directions toward which the original formulation of FBA has been developed in this paper. The algorithm presented here has been conceived to address both these important issues. It represents an extension in respect to algorithms previously proposed to explore the feasible space of solutions and it can also be used to identify the minimal subnetwork(s) able to capture the essence of the whole system in terms of its ability to optimally perform a specific task.

By applying this algorithm on a toy model, we assessed its suitability to achieve these two goals. The algorithm was used firstly to enumerate the alternate optimal patterns of the system, regardless of the number of their active reactions, and secondly to identify its minimal optimal solutions (and hence the subnetworks highlighted by their patterns of active reactions). We note that this second task was performed independently from the first one and that the identification of the minimal optimal solutions can be done directly, without passing through the previous enumeration of the whole set of alternate optimal 
patterns. This would prove particularly useful in the case of a large and highly interconnected network, where finding all the alternate optimal patterns could be extremely time-consuming.

The requirement for an optimal (or suboptimal) solution to have the minimal set of active reactions has previously been adopted by León et al. in a recent study meant to calculate bacterial responses to different growth media (León et al., 2008). For a given set of bounds on the exchange fluxes (defining a specific growth medium) only one solution was found. The approach we propose here has the advantage of combining the minimal effort principle, as stated in the Introduction, with an algorithm able to enumerate the alternate optimal patterns that optimises a given cellular function under a specified set of constraints.

Merging these two features in the same algorithm provides a straightforward way to extend the results of previous studies where the focus on identifying the most "simple" solution in terms of number of active reactions was uncoupled from the problem of enumerating the different patterns in which such a solution can occur.

A second, larger network was then used to compare the results obtained through our algorithm with those presented by Lee et al. (Lee et al., 2000). We found 28 alternate optimal patterns versus the 9 alternate optimal solutions identified in Lee's original work, suggesting our algorithm represents an improvement upon previous approaches proposed to explore the alternatives in the trafficking of matter in metabolic networks. The reason for this higher number of solutions (patterns) lies in the slightly different scope of the two approaches. Here we are looking for different flux distributions in terms of active reactions, regardless of the specific value of the non-zero fluxes. In Lee's work the focus was on finding solutions that were identified by both a specific pattern of active 
reactions and unique values of their fluxes, thus limiting the solutions found to a subset of all the possible alternate optimal patterns.

Further improvements in our approach may be achieved by taking into account the thermodynamics of the system. Currently, the only aspect related to thermodynamic properties is introduced through the upper and lower bounds of the flux variables. Setting one of these bounds to zero, in fact, provides the corresponding reaction with a preferential directionality. Our approach could benefit from considering the thermodynamics of the whole optimal solutions. Following a method proposed by Holzhütter (Holzhütter, 2004), a possible way to do that is by using the equilibrium constants of all the enzymatic steps to weight the fluxes through their corresponding reactions and hence assign an energy cost to the whole solution. This cost would then be used to rank the different solutions by assessing to what extent they are thermodynamically favourable. Although our algorithm is conceived to find alternate optimal patters, which are not necessarily identified by unique sets of fluxes (unless they corresponds to alternate optimal solutions), the implementation of this method does not require any major change to our original algorithm and would provide it with a second criterion to assess the suitability of the solutions in representing the real metabolic phenotype.

\section{Methods}

The central idea of the algorithm proposed in this paper consists in introducing for each flux $v_{j}$ a binary variable $w_{j}^{0}$ which flags the corresponding reaction as active or inactive:

$$
w_{j}^{0}=1 \Leftrightarrow v_{j}=0
$$


In order to make this flagging mechanism work, the general FBA formulation set out in the Introduction (model G.F.) was enriched with a new set of constraints. In particular:

- Every flux variable $v_{j}$ was split into three different variables: $v_{j}^{0}, v_{j}^{-}$and $v_{j}^{+}$.

$$
v_{j}=v_{j}^{0}+v_{j}^{-}+v_{j}^{+}
$$

These new variables are meant to carry respectively the "almost null", negative and positive part of the flux. If flux $v_{j}$ is positive, for example, then $v_{j}^{-}=v_{j}^{0}=0$ and $v_{j}=v_{j}^{+}>0$ (similarly when the flux is negative). If the flux is "reasonably" close to zero to be considered null (i.e. its value is smaller than a given threshold $\varepsilon$ ), then we have $v_{j}^{-}=v_{j}^{+}=0$ and $v_{j}=v_{j}^{0} \simeq 0$.

- For each flux $v_{j}$ three binary variables, $w_{j}^{0}, w_{j}^{-}$and $w_{j}^{+}$, were further introduced in the model. These variables are subject to the following constraint

$$
w_{j}^{0}+w_{j}^{-}+w_{j}^{+}=1
$$

so that only one of them can be non zero (i.e. 1) at the same time. The introduction of these binary variables recast the FBA problem into a mixed integer linear programming (MILP) problem.

- Binary variables $w_{j}^{0}, w_{j}^{-}$and $w_{j}^{+}$were coupled respectively with the previously introduced continuous variables $v_{j}^{0}, v_{j}^{-}$and $v_{j}^{+}$through the following constraints:

$$
\begin{gathered}
v_{j}^{L}\left(1-w_{j}^{0}\right) \leq v_{j} \leq v_{j}^{U}\left(1-w_{j}^{0}\right) \\
-\varepsilon w_{j}^{0}<v_{j}^{0}<\varepsilon w_{j}^{0} \\
v_{j}^{L} w_{j}^{-} \leq v_{j}^{-} \leq \varepsilon w_{j}^{-}
\end{gathered}
$$




$$
\varepsilon w_{j}^{+} \leq v_{j}^{+} \leq w_{j}^{+} v_{j}^{U}
$$

where $\varepsilon$, as mentioned above, is a very small number used as the tolerance for

considering a flux as zero or non-zero (a flux is considered zero when $\left|v_{j}\right|<\varepsilon$ ).

Constraint (5.c) implements the forward implication of equation (4), i.e. when $w_{j}^{0}$ equals 1 the corresponding flux $v_{j}$ is forced to zero.

On the other hand, the backward implication is assured by the constraints (5.d-f) and (5.b) It's easy to prove this statement using a reductio ad absurdum argument. If the flux $v_{j}$ was zero (i.e. $\left|v_{j}\right|<\varepsilon$ ) with $w_{j}^{0}=0$, then either $w_{j}^{+}=1$ or $w_{j}^{-}=1$ (equation 5.b). Let's assume $w_{j}^{+}=1$ (this won't make our argument lose in generality). In this case, $w_{j}^{0}=w_{j}^{-}=0$ (equation 5.b), and consequently $v_{j}^{0}=v_{j}^{-}=0$ (equations 5.d and 5.e). This implies that $v_{j}=v_{j}^{+} \geq \varepsilon$ (equation 5.a and 5.f) contradicting the assumption that $v_{j}$ equals zero (Q.E.D).

The new formulation of the FBA problem can then be written as follows:

$$
\begin{gathered}
\max f(\mathbf{v}) \\
f: \mathbf{R}^{n} \rightarrow \mathbf{R} \\
\text { s.t. }
\end{gathered}
$$

(1), (5.a-f)

The tolerance value $\varepsilon$ used as a threshold to discriminate between zero and non-zero fluxes was set to $10^{-6}$. Model N.F.1 was formulated in GAMS (General Algebraic Modelling System) and solved using the CPLEX solver.

\section{Alternate optimal patterns}


In order to find alternate optimal patterns, the model N.F.1 was run iteratively. At each iteration a new constraint (an integer cut) was added to "cut away" from the feasible space solutions already found in previous iterations. Specifically, at iteration $K$ the following set of integer cuts have been added to the system:

$$
\sum_{j \in Z^{k}} w_{j}^{0}-\sum_{j \in Z^{k}} w_{j}^{0} \leq\left|Z^{k}\right|-1, \quad k=1,2, \ldots, K-1
$$

where $Z^{k}$ is the set of indexes corresponding to the null fluxes (i.e. inactive reactions) at iteration $k,\left|Z^{k}\right|$ denotes its cardinality and $\bar{Z}^{k}$ is the complement of $Z^{k}$ over the whole index set.

More specifically, the algorithm used to find alternate optimal patterns can be described as follows:

\section{Step 1}

a) In the first iteration ( $K=1$ ), model G.F. is solved. The objective function is chosen according to the optimal criterion we want to use.

b) Set $Z^{k}(k=1)$ is defined.

\section{Step $K$, for $K \geq 2$.}

a) Model N.F.1 is solved.

b) Set $Z^{k}$ is defined.

c) If the optimal value of the objective function is not smaller than the value found in the previous iteration the algorithm repeats step $K$, otherwise it stops.

\section{Finding the minimal subnetworks}

To find the minimal subnetwork(s) with the same optimal capability of the whole system, an optimisation was performed using the following formulation: 


$$
\max \sum_{j} w_{j}^{0}
$$

subject to

(1), (5.a-f)

The only difference between N.F.2 and N.F.1 consists in the objective function, here

defined as $f:=\sum_{j} w_{j}^{0}$, i.e. the objective is to maximise the number of reactions with a zero flux. Maximising $f$ forces the solution(s) to have the minimal number of active fluxes. To assure the optimality of the minimal solution(s) found through N.F.2, the general FBA formulation (model G.F.) was first run, in order to find the optimal value of the flux we wanted to optimise; subsequently the flux optimized through G.F. was fixed to the optimal value identified and model N.F.2 was then solved to find the minimal solution(s). As before, model N.F.2 was formulated in GAMS and solved using the CPLEX solver.

\section{Nomenclature}

We present below the complete list of variables and parameters used in the algorithm and their correspondent description

$\begin{array}{ccc}\text { Indices } & i & \text { Index of metabolites } \\ j & \text { Index of reactions } \\ k & \text { Index of iterations }\end{array}$

Sets $\quad Z^{k} \quad$ Set of indexes $j$ corresponding to the null fluxes at iteration $k$.

$\bar{Z}^{k} \quad$ Complement of $Z^{k}$ over the whole index set of the reactions. 
Continuous variables

\begin{tabular}{|c|c|c|}
\hline & $v_{j}^{0}$ & Variable carrying the almost zero part of flux $v_{j}$. \\
\hline & $v_{j}^{-}$ & Variable carrying the negative part of flux $v_{j}$. \\
\hline & $v_{j}^{+}$ & Variable carrying the positive part of flux $v_{j}$. \\
\hline \multirow[t]{3}{*}{ Binary variables } & $w_{j}^{0}$ & Equals 1 if $v_{j}$ is zero, equals 0 otherwise. \\
\hline & $w_{j}^{-}$ & Equals 1 if $v_{j}$ is negative, equals 0 otherwise. \\
\hline & $w_{j}^{+}$ & Equals 1 if $v_{j}$ is positive, equals 0 otherwise. \\
\hline \multirow[t]{4}{*}{ Parameters } & $\mathbf{S}$ & Stoichiometric matrix \\
\hline & $v_{j}^{L}$ & Lower bound of flux $v_{j}$ \\
\hline & $v_{j}^{U}$ & Upper bound of flux $v_{j}$ \\
\hline & $\varepsilon$ & Tolerance for considering a flux as zero or non- \\
\hline
\end{tabular}

$v_{j} \quad$ Variable representing the flux through reaction $j$.

$v_{j}^{0} \quad$ Variable carrying the almost zero part of flux $v_{j}$.

$v_{j}^{-} \quad$ Variable carrying the negative part of flux $v_{j}$.

$v_{j}^{+} \quad$ Variable carrying the positive part of flux $v_{j}$.

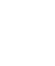




\begin{tabular}{ll|l}
$-r_{1}+r_{16}-r_{18}-r_{17}-r_{31}=0$ & (a.4) & $2.5 r_{13}=0.129$ \\
$r_{1}+r_{18}-r_{21}-r_{19}-r_{20}+r_{32}=0$ & (a.5) & $2.5 r_{15}=1.493$ \\
$-r_{31}-+r_{24}+r_{30}-r_{33}=0$ & (a.6) & $2.5 r_{17}=0.7191$ \\
$r_{21}-r_{24}-r_{23}-r_{22}=0$ & (a.7) & $2.5 r_{6}=0.897$ \\
$r_{24}-r_{25}=0$ & (a.8) & $2.5 r_{9}=0.361$ \\
$r_{25}-r_{27}-r_{26}=0$ & (a.9) & $2.5 r_{20}=2.833$ \\
$-r_{29}+r_{27}-r_{28}=0$ & (a.10) & $2.5 r_{22}=2.928$ \\
$r_{2}-r_{4}-r_{5}=0$ & (a.11) & $2.5 r_{26}=1.078$ \\
$-r_{7}+r_{4}-r_{6}=0$ & (a.12) & $2.5 r_{30}=1.786$ \\
$-r_{7}-r_{8}+r_{5}=0$ & (a.13) & $2 r_{2}+r_{25}+r_{32}=7.2$ \\
$r_{7}-r_{8}-r_{9}=0$ & (a.14) & $r_{\mathrm{ATP}}+r_{12}-3 r_{14}-r_{18}-r_{23}-3 r_{27}$ \\
$2 r_{12}+r_{8}-r_{14}-r_{13}=0$ & (a.15) & $-2 r_{21}-2 r_{33}-r_{29}-r_{31}+2 r_{19}=0$ \\
& &
\end{tabular}

\section{Acknowledgment}

We acknowledge the support of the BBSRC/EPSRC Grant BB/C008219/1 “The Manchester Centre for Integrative Systems Biology" (MCISB). Particular thanks to Lazaros Papageorgiu for the useful discussion. 


\section{References}

Acerenza, L., and Graña, M., 2006. On the Origins of a Crowded Cytoplasm. Journal of Molecular Evolution 63, 583-590.

Beard, D.A., Liang, S.-d., and Qian, H., 2002. Energy Balance for Analysis of Complex Metabolic Networks. Biophysical Journal 83, 79-86.

Beard, D.A., Babson, E., Curtis, E., and Qian, H., 2004. Thermodynamic constraints for biochemical networks. Journal of Theoretical Biology 228, 327-333.

Bonarius, H.P.J., Hatzimanikatis, V., Meesters, K.P.H., Gooijer, C.D.d., Schmid, G., and Tramper, J., 1996. Metabolic Flux Analysis of Hybridoma Cells in Different Culture Media Using Mass Balances. Biotechnology and Bioengineering 50, 299-318.

Cooper, V.S., and Lenski, R.E., 2000. The population genetics of ecological specialization in evolving Escherichia coli populations. Nature 407, 736-739.

Edwards, J.S., and Palsson, B.O., 1999. Systems Properties of the Haemophilus influenzae Rd Metabolic Genotype. Journal of Biological Chemistry 274, 17410-17416.

Edwards, J.S., and Palsson, B.O., 2000. Metabolic flux balance analysis and in silico analysis of Escherichia coli K-12 gene deletions. BMC Bioinformatics 1.

Edwards, J.S., and Palsson, B.O., 2002. The Escherichia coli MG1655 in silico metabolic genotype: Its definition, characteristics, and capabilities. PNAS 97, 5528-5533.

Fell, D.A., and Small, R.J., 1986. Fat synthesis in adipose tissue: an examination of stoichiometric constraints. Biochemical Journal 238, 781-786.

Förster, J., Famili, I., Fu, P., Palsson, B.Ø., and Nielsen, J., 2003. Genome-Scale Reconstruction of the Saccharomyces cerevisiae Metabolic Network. Genome Research 13, 244-253. 
Graña, M., and Acerenza, L., 2001. A model combining cell physiology and population genetics to explain Escherichia coli laboratory evolution. BMC Evolutionary Biology 1.

Holzhütter, H.-G., 2004. The principle of flux minimization and its application to estimate stationary fluxes in metabolic networks. European Journal of Biochemistry 271, $2905-2922$.

Kauffman, K.J., Prakash, P., and Edwards, J.S., 2003. Advances in flux balance analysis. Current Opinion in Biotechnology 14, 491-496.

Lee, J.M., P.Gianchandani, E., and Papin, J.A., 2006. Flux balance analysis in the era of metabolomics. Briefings in Bioinformatics 7, 140-150.

Lee, S., Phalakornkule, C., Domach, M.M., and Grossmann, I.E., 2000. Recursive MILP model for finding all the alternate optima in LP models for metabolic networks. Computers and Chemical Engineering 24, 711-716.

León, M.P.d., Cancela, H., and Acerenza, L., 2008. A Strategy to Calculate the Patterns of Nutrient Consumption by Microorganisms Applying a Two-Level Optimisation Principle to Reconstructed Metabolic Networks. Journal of Biological Physics 34, 73-90.

Llaneras, F., and Picó, J., 2008. Stoichiometric Modelling of Cell Metabolism. Journal of Bioscience and Bioengineering 105, 1-11.

Matheiss, T.H., and Rubin, D.S., 1980. A Survey and Comparison of Methods for Finding All Vertices of Convex Polyhedral Sets. Mathematics of Operations Research 5, 167185.

Price, N.D., Reed, J.L., and Palsson, B.Ø., 2004. Genome-scale models of microbial cells: evaliating the consequences of constraints. Nature 2, 886-897. 
Ramakrishna, R., Edwards, J.S., McCulloch, A., and Palsson, B.O., 2001. Flux-balance analysis of mitochondrial energy metabolism: consequences of systemic stoichiometric constraints. Am J Physiol Regul Integr Comp Physiol 280, 695-704.

Savinell, J.M., and Palsson, B.O., 1992. Network analysis of intermediary metabolism using linear optimization. II. Interpretation of hybridoma cell metabolism. Journal of theoretical biology 154, 455-473.

Schilling, C.H., Letscher, D., and Palsson, B.O., 2000. Theory for the Systemic Definition of Metabolic Pathways and their use in Interpreting Metabolic Function from a Pathway-Oriented Perspective. Journal of Theoretical Biology 203, 229-248.

Schilling, C.H., Covert, M.W., Famili, I., Church, G.M., Edwards, J.S., and Palson, B.O., 2002. Genome-Scale Metabolic Model of Helicobacter pylori 26695. Journal of Bacteriology 184, 4582-4593.

Smallbone, K., and Simeonidis, E., 2009. Flux balance analysis: A geometric perspective. Journal of Theoretical Biology 258, 311-315.

Swart, G., 1985. Finding the convex hull facet by facet. Journal on Algorithms 6, 17-48.

Varma, A., and Palsson, B., 1994. Stoichiometric Flux Balance Models Quantitatively Predict Growth and Metabolic By-Product Secretion in Wild-Type Escherichia coli W3110. Applied and environmental Microbiology 60, 3724-3731.

Varma, A., Boesch, B.W., and Palsson, B.O., 1993. Biochemical Production Capabilities of Escherichia Coli. Biotechnology and Bioengineering 42, 59-73. 